Int. J. Contemp. Math. Sciences, Vol. 2, 2007, no. 18, 891 - 901

\title{
On Functorial Properties of Polynilpotent Multipliers
}

\author{
Mahboobeh Alizadeh Sanati \\ Department of Sciences \\ Golestan University, Gorgan, Iran \\ malizadeh@gau.ac.ir
}

\begin{abstract}
Let $\mathcal{V}$ be a variety of groups. Baer-invariant $\mathcal{V} M(-)$ is a functor from the category of all groups, $\mathcal{G}$ roup, to the category of all abelian groups, $\mathcal{A} b$. In this paper, we show some functional properties of the polynilpotent multiplier of a group $G$ of class row $\left(c_{1}, \ldots, c_{t}\right), \mathcal{N}_{c_{1}, \ldots, c_{t}} M(G)$. In fact, by using some results of the author and others, we try to concentrate on the commutativity of the above functor with the two famous functors Ext and Tor.
\end{abstract}

Mathematics Subject Classification: 20E10, 20K40

Keywords: Baer-invariant, polynilpotent multiplier, Functor, Ext, Tor

\section{Introduction}

Let $G$ be a group with a free presentation

$$
1 \longrightarrow R \longrightarrow F \longrightarrow G \longrightarrow 1
$$

where $F$ is a free group and $R$ a normal subgroup of $F$ such that the above sequence is exact. Then the Baer-invariant of $G$, after R. Baer [1], with respect 
to the variety $\mathcal{V}$, denoted by $\mathcal{V} M(G)$, is defined to be

$$
\mathcal{V} M(G)=\frac{R \cap V(F)}{V(R, F)}
$$

where $V(F)$ is the verbal subgroup of $F$ with respect to $\mathcal{V}$, as

$$
\left\{v\left(f_{1}, \cdots, \cdots, f_{s}\right) \mid v \in V, f_{i} \in F, 1 \leq i \leq s\right\}
$$

and

$$
\begin{gathered}
V(R, F)=<v\left(f_{1}, \ldots, f_{i-1}, f_{i} r, f_{i+1}, \ldots, f_{n}\right) v\left(f_{1}, \ldots, f_{i}, \ldots f_{n}\right)^{-1} \mid r \in R \\
1 \leq i \leq n, v \in V, f_{i} \in F, n \in \mathbf{N}>
\end{gathered}
$$

It can be proved that the Baer-invariant of a group $G$ is independent of the choice of the presentation of $G$ and it is always an abelian group (See [7]).

In particular, if $\mathcal{V}$ is the variety of abelian groups, $\mathcal{A}$, then the Baerinvariant of $G$ will be $\left(R \cap F^{\prime}\right) /[R, F]$, which, following Hopf [5], is isomorphic to the second cohomology group of $G, H_{2}\left(G, \mathbf{C}^{*}\right)$, in finite case, and also is isomorphic to the well-known notion the Schur multiplier of $G$, denoted by $M(G)($ see $[14,15]$.

If $\mathcal{V}$ is the variety of nilpotent groups of class at most $c \geq 1, \mathcal{N}_{c}$, then the Baer-invariant of the group $G$ will be

$$
\mathcal{N}_{c} M(G)=\frac{R \cap \gamma_{c+1}(F)}{\left[R,{ }_{c} F\right]},
$$

where $\gamma_{c+1}(F)$ is the $(c+1)$ st term of the lower central series of $F$ and $\left[R,_{1} F\right]=$ $[R, F],\left[R,_{c} F\right]=\left[\left[R,_{c-1} F\right], F\right]$, inductively. The above notion is also called the $c$-nilpotent multiplier of $G$ and denoted by $M^{(c)}(G)$ (see [7]).

If $\mathcal{V}=\mathcal{N}_{c_{1}, \ldots, c_{t}}$, the variety of polynilpotent groups of class row $\left(c_{1}, \ldots, c_{t}\right)$, then

$$
\mathcal{N}_{c_{1}, \ldots, c_{t}} M(G)=\frac{R \cap \gamma_{c_{t}+1} \circ \cdots \circ \gamma_{c_{1}+1}(F)}{\left[R, c_{1} F_{c_{2}} \gamma_{c_{1}+1}(F), \cdots,{ }_{c_{t}} \gamma_{c_{t-1}+1} \circ \cdots \circ \gamma_{c_{1}+1}(F)\right]},
$$

where $\gamma_{c_{t}+1} \circ \cdots \circ \gamma_{c_{1}+1}(F)=\gamma_{c_{t}+1}\left(\gamma_{c_{t-1}+1}\left(\cdots\left(\gamma_{c_{1}+1}(F)\right) \cdots\right)\right)$ are the terms of iterated lower central series of $F$. See [4, corollary 6.14] for the following equality

$$
N_{c_{1}, \ldots, c_{t}}(R, F)=\left[R,_{c_{1}} F{, c_{2}}_{c_{1}+1}(F), \ldots,,_{t} \gamma_{c_{t-1}+1} \circ \ldots \circ \gamma_{c_{1}+1}(F)\right]
$$


We shall also call $\mathcal{N}_{c_{1}, \ldots, c_{t}} M(G)$, the $\left(c_{1}, \ldots, c_{t}\right)$-polynilpotent multiplier of $G$.

\section{Definition $1.1[2]$}

The notion of basic commutators on letters $x_{1}, x_{2}, \ldots, x_{n}, \ldots$, are defined as follows:

(i) The letters $x_{1}, x_{2}, \ldots, x_{n}, \ldots$ are basic commutators of weight one, ordered by setting $x_{i}<x_{j}$ if $i<j$.

(ii) If basic commutators $c_{i}$ of weight $w t\left(c_{i}\right)<k$ are defined and ordered, then define basic commutators of weight $k$ by the following rules:

$\left[c_{i}, c_{j}\right]$ is a basic commutator of weight $k$ if

1. $w t\left(c_{i}\right)+w t\left(c_{j}\right)=k$,

2. $c_{i}>c_{j}$,

3. if $c_{i}=\left[c_{s}, c_{t}\right]$, then $c_{j} \geq c_{t}$.

Then continue the order by setting $c>c_{i}$ whenever $w t(c)>w t\left(c_{i}\right)$ and fixing any order amongst those of weight $k$ and finally numbering them in order.

Theorem 1.2 (P.Hall $[2,3])$

Let $F=<x_{1}, x_{2}, \ldots, x_{d}>$ be a free group, then

$$
\frac{\gamma_{n}(F)}{\gamma_{n+i}(F)}, \quad 1 \leq i \leq n
$$

is the free abelian group freely generated by the basic commutators of weights $n, n+1, \ldots, n+i-1$ on the letters $\left\{x_{1}, \ldots, x_{d}\right\}$.

\section{Theorem 1.3 (Witt Formula [3])}

The number of basic commutators of weight $n$ on $d$ generators is given by the following formula:

$$
\chi_{n}(d)=\frac{1}{n} \sum_{m \mid n} \mu(m) d^{n / m}
$$

where $\mu(m)$ is the Mobious function, and defined to be

$$
\mu(m)= \begin{cases}1 & \text { if } m=1 \\ 0 & \text { if } m=p_{1}^{\alpha_{1}} \ldots p_{k}^{\alpha_{k}} \quad \exists \alpha_{i}>1 \\ (-1)^{s} & \text { if } m=p_{1} \ldots p_{s}\end{cases}
$$


Our main results are as follows that they are the generalization of [9].

Theorem A. Let $\mathcal{N}_{c_{1}, c_{2}, \ldots, c_{t}}$ be the polynilpotent variety of class row $\left(c_{1}, c_{2}, \ldots, c_{t}\right)$ and $G$ be a finitely generated abelian group. Then for all $n \geq 0$

$$
\mathcal{N}_{c_{1}, c_{2}, \ldots, c_{t}} M\left(\operatorname{Ext}_{\mathbf{Z}}^{n}\left(\mathbf{Z}_{m}, G\right)\right) \cong \operatorname{Ext}_{\mathbf{Z}}^{n}\left(\mathbf{Z}_{m}, \mathcal{N}_{c_{1}, c_{2}, \ldots, c_{t}} M(G)\right)
$$

If $G$ is finite,

$$
\mathcal{N}_{c_{1}, c_{2}, \ldots, c_{t}} M\left(\operatorname{Ext}_{\mathbf{Z}}^{n}\left(G, \mathbf{Z}_{m}\right)\right) \cong \operatorname{Ext}_{\mathbf{Z}}^{n}\left(\mathcal{N}_{c_{1}, c_{2}, \ldots, c_{t}} M(G), \mathbf{Z}_{m}\right)
$$

Theorem B. If $G$ is a finite abelian group and $\mathcal{N}_{c_{1}, c_{2}, \ldots, c_{t}}$ be the polynilpotent variety of class row $\left(c_{1}, c_{2}, \ldots, c_{t}\right)$, then for all $n \geq 0$

$$
\begin{aligned}
& (i) \mathcal{N}_{c_{1}, c_{2}, \ldots, c_{t}} M\left(\operatorname{Tor}_{n}^{\mathbf{Z}}\left(\mathbf{Z}_{m}, G\right)\right) \cong \operatorname{Tor}_{n}^{\mathbf{Z}}\left(\mathbf{Z}_{m}, \mathcal{N}_{c_{1}, c_{2}, \ldots, c_{t}} M(G)\right) \\
& (i i) \mathcal{N}_{c_{1}, c_{2}, \ldots, c_{t}} M\left(\operatorname{Tor}_{n}^{\mathbf{Z}}\left(G, \mathbf{Z}_{m}\right)\right) \cong \operatorname{Tor}_{n}^{\mathbf{Z}}\left(\mathcal{N}_{c_{1}, c_{2}, \ldots, c_{t}} M(G), \mathbf{Z}_{m}\right)
\end{aligned}
$$

\section{Elementary Results}

The following theorem permits us to look at the notion of the Baer-invariant as a functor.

\section{Theorem 2.1}

Let $\mathcal{V}$ be an arbitrary variety of groups. Then, using the notion of the Baerinvariant, we can consider the following covariant functor from the category of all groups, $\mathcal{G}$ roup, to the category of all abelian groups, $\mathcal{A} b$

$$
\mathcal{V} M(-): \mathcal{G r o u p} \longrightarrow \mathcal{A} b
$$

which assigns to any group $G$ the abelian group $\mathcal{V} M(G)$.

Proof. See [7].

In [8] have been shown that the Baer-invariant functor is not additive nor right and left exact even if we restrict ourself to abelian groups.

In 1952, C. Miller [13] proved that the Schur multiplier of a free product is isomorphic to the direct sum of the Schur multipliers of the free factors. In other words, he proved that the Schur multiplier functor $M(-)$ is 
coproduct-preserving. But the second nilpotent multiplier functor, $\mathcal{N}_{2} M(-)$, is not coproduct preserving, in general [8]. Also, in 1980 M.R.R. Moghaddam [12] proved that in general, the Baer-invariant functor commutes with direct limit of a directed system of groups.

Theorem 2.2 (M.R.R. Moghaddam [12])

Let $\left\{G_{i} ; \alpha_{i}^{j}, I\right\}$ be a direct system of groups and $\mathcal{V}$ an arbitrary variety of groups. Then

$$
\mathcal{V} M\left(\stackrel{\lim }{\rightarrow} G_{i}\right) \cong \stackrel{\lim }{\rightarrow} \mathcal{V} M\left(G_{i}\right)
$$

In the rest, we need an explicit formula for the polynilpotent multiplier of a finitely generated abelian groups which is recently proved by B. Mashayekhy and M. Parvizi as follows.

\section{Theorem 2.3 (B. Mashayekhy and M. Parvizi [11])}

Let $\mathcal{N}_{c_{1}, c_{2}, \ldots, c_{t}}$ be the polynilpotent variety of class row $\left(c_{1}, c_{2}, \ldots, c_{t}\right)$ and $G \cong \mathbf{Z}^{(n)} \oplus \mathbf{Z}_{n_{1}} \oplus \ldots \oplus \mathbf{Z}_{n_{k}}$ be a finitely generated abelian group, where $n_{i+1} \mid n_{i}$ for all $1 \leq 1 \leq k-1$. Then an explicit structure of the polynilpotent multiplier of $G$ is as follows

$$
\mathcal{N}_{c_{1}, c_{2}, \ldots, c_{t}} M(G) \cong \mathbf{Z}^{\left(f_{n}\right)} \oplus \mathbf{Z}_{n_{1}}^{\left(f_{n+1}-f_{n}\right)} \oplus \ldots \oplus \mathbf{Z}_{n_{k}}^{\left(f_{n+k}-f_{n+k-1}\right)}
$$

where $f_{i}=\chi_{c_{t}+1}\left(\chi_{c_{t-1}+1}\left(\ldots\left(\chi_{c_{1}+1}(i)\right) \ldots\right)\right)$ for all $n \leq i \leq n+k$.

In Theorem 2.3 consider $t=1$ and $n=0$, then we immediately conclude the following theorem which it is obtained in 1997 as follows.

Theorem 2.4 (B. Mashayekhy and M.R.R. Moghaddam [10])

Let $G \cong \mathbf{Z}_{n_{1}} \oplus \mathbf{Z}_{n_{2}} \oplus \ldots \oplus \mathbf{Z}_{n_{k}}$, be a finite abelian group, where $n_{i+1} \mid n_{i}$ for all $1 \leq i \leq k-1$ and $k \geq 2$. Then, for all $c \geq 1$, the $c$-nilpotent multiplier of $G$ is

$$
\mathcal{N}_{c} M(G) \cong \mathbf{Z}_{n_{2}}^{\left(\chi_{c_{1}+1}(2)\right)} \oplus \mathbf{Z}_{n_{3}}^{\left(\chi_{c_{1}+1}(3)-\chi_{c_{1}+1}(2)\right)} \oplus \ldots \oplus \mathbf{Z}_{n_{k}}^{\left(\chi_{c_{1}+1}(k)-\chi_{c_{1}+1}(k-1)\right)} .
$$

By using Theorem 2.4, one can see that the $c$-nilpotent multiplier functors can preserve every elementary abelian $p$-group. 


\section{Main Results}

In this section, we will see the behaviour of the functor $\left.\mathcal{N}_{(} c_{1}, \ldots, c_{t}\right) M(-)$ with the functors $\operatorname{Ext}_{\mathbf{Z}}^{n}(A,-), \operatorname{Ext}_{\mathbf{Z}}^{n}(-, A)$ and $\operatorname{Tor}_{n}^{\mathbf{Z}}(A,-)$. We know for all $n \geq 2$, $\operatorname{Tor}_{n}^{\mathbf{Z}}(A, B)=0$ and $\operatorname{Ext}_{\mathbf{Z}}^{n}(A, B)=0$ (see [16]). So, we concentrate ourselves on $n=0,1$. First, we need the following lemmas.

\section{Lemma 3.1}

For any abelian groups $A$ and $B$, we have

(i) $\operatorname{Ext}_{\mathbf{Z}}^{1}(\mathbf{Z} / m \mathbf{Z}, B) \cong B / m B$.

(ii) If $A$ and $B$ are finite abelian groups, then

$$
\operatorname{Ext}_{\mathbf{Z}}^{1}(A, B) \cong \operatorname{Ext}_{\mathbf{Z}}^{1}(B, A) .
$$

(iii) $\operatorname{Tor}_{1}^{\mathbf{Z}}(\mathbf{Z} / m \mathbf{Z}, B) \cong B[m]$, where $B[m]=\{b \in B: m b=0\}$.

(iv) $\operatorname{Tor}_{1}^{\mathbf{Z}}(A, B) \cong \operatorname{Tor}_{1}^{\mathbf{Z}}(B, A)$.

Proof. See [16, Chapters 7, 8].

\section{Lemma 3.2}

Let $A$ and $\left\{B_{k}\right\}_{k \in I}$ be abelian groups. Then for all $n \geq 0$ the following isomorphism hold.

(i) $\operatorname{Ext}_{\mathbf{Z}}^{n}\left(A, \prod_{k \in I} B_{k}\right) \cong \prod_{k \in I} \operatorname{Ext}_{\mathbf{Z}}^{n}\left(A, B_{k}\right), \operatorname{Ext}_{\mathbf{Z}}^{n}\left(\coprod_{k \in I} B_{k}, A\right) \cong \prod_{k \in I} \operatorname{Ext}_{\mathbf{Z}}^{n}\left(B_{k}, A\right)$.

(ii) $\operatorname{Tor}_{n}^{\mathbf{Z}}\left(A, \coprod_{k \in I} B_{k}\right) \cong \coprod_{k \in I} \operatorname{Tor}_{n}^{\mathbf{Z}}\left(A, B_{k}\right), \operatorname{Tor}_{n}^{\mathbf{Z}}\left(\coprod_{k \in I} B_{k}, A\right) \cong \coprod_{k \in I} \operatorname{Tor}_{n}^{\mathbf{Z}}\left(B_{k}, A\right)$.

Proof. See [16].

It is obvious that the functor $\mathcal{N}_{c_{1}, c_{2}, \ldots, c_{t}} M(-)$ commutes with the functors $\operatorname{Ext}_{\mathbf{Z}}^{n}\left(\mathbf{Z}_{m},-\right)$, and $\operatorname{Tor}_{n}^{\mathbf{Z}}\left(\mathbf{Z}_{m},-\right)$ for all $n \geq 2$, by lemma 3.2. Now we are going to pay our attention to the functors of $\operatorname{Ext}_{\mathbf{Z}}^{1}\left(\mathbf{Z}_{m},-\right), \operatorname{Ext}_{\mathbf{Z}}^{1}\left(-, \mathbf{Z}_{m}\right)$ and $\operatorname{Tor}_{1}^{\mathbf{Z}}\left(\mathbf{Z}_{m},-\right)$.

\section{Theorem 3.3}

Let $G \cong \mathbf{Z}^{(n)} \oplus \mathbf{Z}_{n_{1}} \oplus \mathbf{Z}_{n_{2}} \oplus \ldots \oplus \mathbf{Z}_{n_{k}}$, be a finitely generated abelian group, where $n \geq 0, n_{i+1} \mid n_{i}$ for all $1 \leq i \leq k-1$. Then, for all $\left(c_{1}, c_{2}, \ldots, c_{t}\right)$, the following isomorphisms hold.

(i) $\mathcal{N}_{c_{1}, c_{2}, \ldots, c_{t}} M\left(\operatorname{Ext}_{\mathbf{Z}}^{1}\left(\mathbf{Z}_{m}, G\right)\right) \cong \mathbf{Z}_{m}^{\left(f_{n}\right)} \oplus\left(\oplus \sum_{i=1}^{k} \mathbf{Z}_{\left(m, n_{i}\right)}^{\left(f_{n+i}-f_{n+i-1}\right)}\right)$. 
(ii) $\operatorname{Ext}_{\mathbf{Z}}^{1}\left(\mathbf{Z}_{m}, \mathcal{N}_{c_{1}, c_{2}, \ldots, c_{t}} M(G)\right) \cong \mathbf{Z}_{m}^{\left(f_{n}\right)} \oplus\left(\oplus \sum_{i=1}^{k} \mathbf{Z}_{\left(m, n_{i}\right)}^{\left(f_{n+i}-f_{n+i-1}\right)}\right)$.

(iii) $\mathcal{N}_{c_{1}, c_{2}, \ldots, c_{t}} M\left(\operatorname{Ext}_{\mathbf{Z}}^{1}\left(G, \mathbf{Z}_{m}\right)\right) \cong \oplus \sum_{i=2}^{k} \mathbf{Z}_{\left(m, n_{i}\right)}^{\left(f_{i}-f_{i-1}\right)}$.

(iv) $\operatorname{Ext}_{\mathbf{Z}}^{1}\left(\mathcal{N}_{c_{1}, c_{2}, \ldots, c_{t}} M(G), \mathbf{Z}_{m}\right) \cong \oplus \sum_{i=1}^{k} \mathbf{Z}_{\left(m, n_{i}\right)}^{\left(f_{n+i}-f_{n+i-1}\right)}$.

(v) $\mathcal{N}_{c_{1}, c_{2}, \ldots, c_{t}} M\left(\operatorname{Tor}_{1}^{\mathbf{Z}}\left(\mathbf{Z}_{m}, G\right)\right) \cong \oplus \sum_{i=2}^{k} \mathbf{Z}_{\left(m, n_{i}\right)}^{\left(f_{i}-f_{i-1}\right)}$.

(vi) $\operatorname{Tor}_{1}^{\mathbf{Z}}\left(\mathbf{Z}_{m}, \mathcal{N}_{c_{1}, c_{2}, \ldots, c_{t}} M(G)\right) \cong \oplus \sum_{i=1}^{k} \mathbf{Z}_{\left(m, n_{i}\right)}^{\left(f_{n+i}-f_{n+i-1}\right)}$.

Proof. (i) By Lemma 3.3(i), $\operatorname{Ext}_{\mathbf{Z}}^{1}(\mathbf{Z} / m \mathbf{Z}, \mathbf{Z}) \cong \mathbf{Z} / m \mathbf{Z} \cong \mathbf{Z}_{m}$. Now by using Lemmas 3.3(i) and 3.2(i), we have

$$
\begin{gathered}
\operatorname{Ext}_{\mathbf{Z}}^{1}\left(\mathbf{Z}_{m}, G\right) \cong\left(\operatorname{Ext}_{\mathbf{Z}}^{1}\left(\mathbf{Z}_{m}, \mathbf{Z}\right)\right)^{(n)} \oplus\left(\oplus \sum_{i=1}^{k} \operatorname{Ext}_{\mathbf{Z}}^{1}\left(\mathbf{Z}_{m}, \mathbf{Z}_{n_{i}}\right)\right) \\
\cong \mathbf{Z}_{m}^{(n)} \oplus\left(\oplus \sum_{i=1}^{k} \mathbf{Z}_{n_{i}} / m \mathbf{Z}_{n_{i}}\right) \cong \mathbf{Z}_{m}^{(n)} \oplus\left(\oplus \sum_{i=1}^{k} \mathbf{Z}_{\left(m, n_{i}\right)}\right)
\end{gathered}
$$

Now, by Theorem 2.2 and noting that $\left(m, n_{i+1}\right)\left|\left(m, n_{i}\right)\right| m$ we have

$$
\begin{gathered}
\mathcal{N}_{c_{1}, c_{2}, \ldots, c_{t}} M\left(\operatorname{Ext}_{\mathbf{Z}}^{1}\left(\mathbf{Z}_{m}, G\right)\right) \\
\cong \mathbf{Z}_{m}^{\left(f_{2}-f_{1}\right)} \oplus \mathbf{Z}_{m}^{\left(f_{3}-f_{2}\right)} \oplus \ldots \oplus \mathbf{Z}_{m}^{\left(f_{n}-f_{n-1}\right)} \oplus \mathbf{Z}_{\left(m, n_{1}\right)}^{\left(f_{n+1}-f_{n}\right)} \oplus \ldots \oplus \mathbf{Z}_{\left(m, n_{k}\right)}^{\left(f_{n+k}-f_{n+k-1}\right)} \\
\cong \mathbf{Z}_{m}^{\left(f_{n}\right)} \oplus\left(\oplus \sum_{i=1}^{k} \mathbf{Z}_{\left(m, n_{i}\right)}^{\left(f_{n+i}-f_{n+i-1}\right)}\right) .
\end{gathered}
$$

(ii) By Theorem 3.1 and Lemmas 3.3(i) and 3.2(i), we have

$$
\begin{gathered}
\operatorname{Ext}_{\mathbf{Z}}^{1}\left(\mathbf{Z}_{m}, \mathcal{N}_{c_{1}, c_{2}, \ldots, c_{t}} M(G)\right) \\
\cong\left(\operatorname{Ext}_{\mathbf{Z}}^{1}\left(\mathbf{Z}_{m}, \mathbf{Z}\right)\right)^{\left(f_{n}\right)} \oplus\left(\oplus \sum_{i=1}^{k}\left(\operatorname{Ext}_{\mathbf{Z}}^{1}\left(\mathbf{Z}_{m}, \mathbf{Z}_{n_{i}}\right)\right)^{\left(f_{n+i}-f_{n+i-1}\right)}\right) \\
\cong \mathbf{Z}_{m}^{\left(f_{n}\right)} \oplus\left(\oplus \sum_{i=1}^{k} \mathbf{Z}_{\left(m, n_{i}\right)}^{\left(f_{n+i}-f_{n+i-1}\right)}\right)
\end{gathered}
$$

(iii) and (iv) By similar methods of $(i)$ and (ii).

$(v)$ By Lemmas 3.3(ii) and 3.2(ii) we have

$$
\operatorname{Tor}_{1}^{\mathbf{Z}}\left(\mathbf{Z}_{m}, G\right) \cong\left(\operatorname{Tor}_{1}^{\mathbf{Z}}\left(\mathbf{Z}_{m}, \mathbf{Z}\right)\right)^{(n)} \oplus\left(\oplus \sum_{i=1}^{k} \operatorname{Tor}_{1}^{\mathbf{Z}}\left(\mathbf{Z}_{m}, \mathbf{Z}_{n_{i}}\right)\right) \cong \oplus \sum_{i=1}^{k} \mathbf{Z}_{n_{i}}[m] .
$$

Note that $\operatorname{Tor}_{1}^{\mathbf{Z}}\left(\mathbf{Z}_{m}, \mathbf{Z}\right) \cong 1$ and $\mathbf{Z}_{n}[m] \cong \mathbf{Z}_{(m, n)}$. So we have $\operatorname{Tor}_{1}^{\mathbf{Z}}\left(\mathbf{Z}_{m}, G\right) \cong$ $\oplus \sum_{i=1}^{k} \mathbf{Z}_{\left(m, n_{i}\right)}$. Now by Theorem 2.2 the result holds. 
(vi) Again by using Theorem 3.1 and Lemmas 3.3(ii) and 3.2(ii), we have

$$
\begin{aligned}
\operatorname{Tor}_{1}^{\mathbf{Z}}\left(\mathbf{Z}_{m}, \mathcal{N}_{c_{1}, c_{2}, \ldots, c_{t}} M(G)\right) & \cong\left(\operatorname{Tor}_{1}^{\mathbf{Z}}\left(\mathbf{Z}_{m}, \mathbf{Z}\right)\right)^{\left(f_{n}\right)} \oplus\left(\oplus \sum_{i=1}^{k} \operatorname{Tor}_{1}^{\mathbf{Z}}\left(\mathbf{Z}_{m}, \mathbf{Z}_{n_{i}}^{\left(f_{n+i}-f_{n+i-1}\right)}\right)\right. \\
& \cong \oplus \sum_{i=1}^{k} \mathbf{Z}_{\left(m, n_{i}\right)}^{\left(f_{n+i}-f_{n+i-1}\right)} .
\end{aligned}
$$

This theorem means that the polynilpotent multiplier functors of class row $c_{1}, c_{2}, \ldots, c_{t}, \mathcal{N}_{c_{1}, c_{2}, \ldots, c t} M(-)$ do not commute with $\operatorname{Tor}_{1}^{\mathbf{Z}}\left(\mathbf{Z}_{m},-\right)$ and $\operatorname{Ext}_{\mathbf{Z}}^{1}\left(-, \mathbf{Z}_{m}\right)$ in infinite case.

Now you can find the proof of main results of the paper.

Proof A. (i) It is clear by parts $(i)$, (ii) of the previous theorem. (ii) The parts $(i i i)$ and $(i v)$ of the previous theorem conclude the result.

Proof B. Since $G$ is infinite, so $n \geq 1$. Hence the result holds by the previous theorem parts $(v)$ and $(v i)$. The second part is similar to first one.

We know that $\operatorname{Hom}\left(\mathbf{Z}_{m}, \mathbf{Z}\right) \cong 1$ and $\operatorname{Hom}\left(\mathbf{Z}, \mathbf{Z}_{m}\right) \cong \mathbf{Z}_{m}$. So by similar methods of Theorem 3.4 we are going to look at the behaviour of functor $\mathcal{N}_{c_{1}, c_{2}, \ldots, c_{t}} M(-)$ with $\operatorname{Ext} t_{\mathbf{Z}}^{0}\left(\mathbf{Z}_{m},-\right)=\operatorname{Hom}\left(\mathbf{Z}_{m},-\right), \operatorname{Ext} t_{\mathbf{Z}}^{0}\left(-, \mathbf{Z}_{m}\right)=\operatorname{Hom}\left(-, \mathbf{Z}_{m}\right)$ and $\operatorname{Tor}_{0}^{\mathbf{Z}}\left(\mathbf{Z}_{m},-\right)=\mathbf{Z}_{m} \otimes-$ as the following theorem.

\section{Theorem 3.5}

For any finitely generated abelian group $G \cong \mathbf{Z}^{(n)} \oplus \mathbf{Z}_{n_{1}} \oplus \mathbf{Z}_{n_{2}} \oplus \ldots \oplus \mathbf{Z}_{n_{k}}$, we have

(i) $\mathcal{N}_{c_{1}, c_{2}, \ldots, c_{t}} M\left(\operatorname{Hom}\left(\mathbf{Z}_{m}, G\right) \cong \mathbf{Z}_{\left(m, n_{2}\right)}^{\left(f_{2}\right)} \oplus \ldots \oplus \mathbf{Z}_{\left(m, n_{k}\right)}^{\left(f_{k}-f_{k-1}\right)}\right.$.

(ii) $\operatorname{Hom}\left(\mathbf{Z}_{m}, \mathcal{N}_{c_{1}, c_{2}, \ldots, c_{t}} M(G)\right) \cong \mathbf{Z}_{\left(m, n_{1}\right)}^{\left(f_{n+1}-f_{n}\right)} \oplus \ldots \oplus \mathbf{Z}_{\left(m, n_{k}\right)}^{\left(f_{n+k}-f_{n+k-1}\right)}$.

(iii)If $G$ is finite, then $\mathcal{N}_{c_{1}, c_{2}, \ldots, c_{t}} M\left(\operatorname{Hom}\left(\mathbf{Z}_{m}, G\right) \cong \operatorname{Hom}\left(\mathbf{Z}_{m}, \mathcal{N}_{c_{1}, c_{2}, \ldots, c_{t}} M(G)\right)\right.$. If $G$ is infinite, then $\mathcal{N}_{c_{1}, c_{2}, \ldots, c_{t}} M\left(\operatorname{Hom}\left(\mathbf{Z}_{m}, G\right) \not \operatorname{Hom}\left(\mathbf{Z}_{m}, \mathcal{N}_{c_{1}, c_{2}, \ldots, c_{t}} M(G)\right)\right.$. (iv)

$$
\mathcal{N}_{c_{1}, c_{2}, \ldots, c_{t}} M\left(\operatorname{Hom}\left(G, \mathbf{Z}_{m}\right)\right) \cong \operatorname{Hom}\left(\mathcal{N}_{c_{1}, c_{2}, \ldots, c_{t}} M(G), \mathbf{Z}_{m}\right)
$$




$$
\cong \mathbf{Z}_{m}^{\left(f_{n}\right)} \oplus \mathbf{Z}_{\left(m, n_{1}\right)}^{\left(f_{n+1}-f_{n}\right)} \oplus \ldots \oplus \mathbf{Z}_{\left(m, n_{k}\right)}^{\left(f_{n+k}-f_{n+k-1}\right)}
$$

$(v)$

$$
\begin{gathered}
\mathcal{N}_{c_{1}, c_{2}, \ldots, c_{t}} M\left(\mathbf{Z}_{m} \otimes G\right) \cong \mathbf{Z}_{m} \otimes \mathcal{N}_{c_{1}, c_{2}, \ldots, c_{t}} M(G) \\
\cong \mathbf{Z}_{m}^{\left(f_{n}\right)} \oplus \mathbf{Z}_{\left(m, n_{1}\right)}^{\left(f_{n+1}-f_{n}\right)} \oplus \ldots \oplus \mathbf{Z}_{\left(m, n_{k}\right)}^{\left(f_{n+k}-f_{n+k-1}\right)}
\end{gathered}
$$

Now, in the following we are going to show that our conditions in the previous results are essential. In general case $\operatorname{Ext}_{\mathbf{Z}}^{i}(A,-), \operatorname{Ext}_{\mathbf{Z}}^{i}(-, A)$ and $\operatorname{Tor}_{i}^{\mathbf{Z}}(A,-)$, where $A$ is not cyclic, do not commute with $\mathcal{N}_{c_{1}, c_{2}, \ldots, c_{t}} M(-)$, for $i=0,1$.

Example $(i)$. Let $A \cong \mathbf{Z}_{r} \oplus \mathbf{Z}_{s}$ and $G \cong \mathbf{Z}_{m} \oplus \mathbf{Z}_{n}$, where $n \mid m$ and $m \mid(r, s)$. Then

$\mathcal{N}_{c_{1}, c_{2}, \ldots, c_{t}} M\left(\operatorname{Ext}_{\mathbf{Z}}^{1}(A, G) \cong \mathbf{Z}_{m}^{\left(f_{2}\right)} \oplus \mathbf{Z}_{n}^{\left(f_{4}-f_{2}\right)} ¥ \mathbf{Z}_{n}^{\left(2 f_{2}\right)} \cong \operatorname{Ext}_{\mathbf{Z}}^{1}\left(A, \mathcal{N}_{c_{1}, c_{2}, \ldots, c_{t}} M(G)\right)\right.$

i.e the functor $\mathcal{N}_{c_{1}, c_{2}, \ldots, c_{t}} M(-)$ does not commute with $\operatorname{Ext}_{\mathbf{Z}}^{1}(A,-)$. Similarly the polynilpotent functor does not commute with the following functors: $\operatorname{Ext}_{\mathbf{Z}}^{i}(A,-), \operatorname{Ext}_{\mathbf{Z}}^{i}(-, A), \operatorname{Tor}_{i}^{\mathbf{Z}}(A,-)$ for $i=0,1$.

Example $(i i) \cdot \operatorname{Hom}\left(S_{n}, \mathbf{Z}_{2}\right) \cong \mathbf{Z}_{2}$, for $n \geq 2$. Also we know that $M\left(S_{n}\right) \cong$ $\mathbf{Z}_{2}$, for each $n \geq 4$, see [6, theorem 2.12.3]. Thus

$$
\begin{gathered}
M\left(\operatorname{Hom}\left(\mathbf{Z}_{2}, S_{n}\right)\right) \cong M\left(\operatorname{Hom}\left(S_{n}, \mathbf{Z}_{2}\right)\right) \cong 1 \\
¥ \mathbf{Z}_{2} \cong \operatorname{Hom}\left(M\left(S_{n}\right), \mathbf{Z}_{2}\right) \cong \operatorname{Hom}\left(\mathbf{Z}_{2}, M\left(S_{n}\right)\right) . \\
\left.M\left(S_{n} \otimes \mathbf{Z}_{2}\right)\right) \cong 1 \nsubseteq \mathbf{Z}_{2} \cong M\left(S_{n}\right) \otimes \mathbf{Z}_{2} .
\end{gathered}
$$

\section{References}

[1] R. Baer, Representations of groups as quotient groups, I-III Trans. Amer. Math. Soc, 58 (1945), 295 -419.

[2] P. Hall, The classification of prime power groups, J. Reine Angew. Math, 182 (1940), 130 -141. 
[3] P. Hall, Nilpotent Groups, Cand. Math. Congress, Univ. of Alberta (1957). Queen Mary College Math. Notes, London (1970).

[4] N.S. Hekster, Varieties of groups and isologisms, J. Austral. Math. Soc. (Series A) 46 (1989), 22 -60.

[5] H. Hopf, Fundamental gruppe und zweite bettische gruppe, Comment. Math. Helvetici 14 (1942), 257 -309.

[6] G. Karpilovsky, The Schur Multiplier, London Math. Soc. Monographs (N.S.), Vol. 2, Oxford Univ. Press, London (1987).

[7] C. R. Leedham-Green and S. McKay, Baer-invariant, isologism, varietal laws and homology, Acta Math. 137 (1976), 99 -150.

[8] B. Mashayekhy, On the nilpotent multiplier of a free product, Bull. Iran Math. Soc. 28:2 (2002), 49 -56.

[9] B. Mashayekhy and M. Alizadeh Sanati, Some Functorial Properties of Nilpotent Multipliers, International J. of Mathematics Game Theory and Algebra, 13:2 (2003), 129 -132.

[10] B. Mashayekhy and M. R. R. Moghaddam, Higher Schur multiplicator of a finite abelian group, Algebra Colloquium 4:3 (1997), 317 -322.

[11] B. Mashayekhy and M. Parvizi, Polynilpotent Multipliers of Finitely Generated Abelian Groups, International Journal of Mathematics, Game Theory and Algebra 16:2 (2006), 93 -102.

[12] M. R. R. Moghaddam, The Baer-invariant and the direct limit, Mh. Math. 90 (1980), $37-43$.

[13] C. Miller, The second homology group of a group; relation among commutators, Proc. Amer. Math. Soc. 3 (1952), 588 -95.

[14] I. Schur, Über die darstellung der endlichen gruppen durch gebrochene lineare substitutionen, J. Reine Angew. Math. 127 (1904), 20 -50. 
[15] I. Schur, Untersuchungen über die darstellung der endlichen gruppen durch gebrochene lineare substitutionen, J. Reine Angew. Math. 132 (1907), $85-137$.

[16] L.R. Vermani, An Elementary Approach to Homological Algebra, New York, A CRC Press Company (2003).

Received: January 7, 2007 